
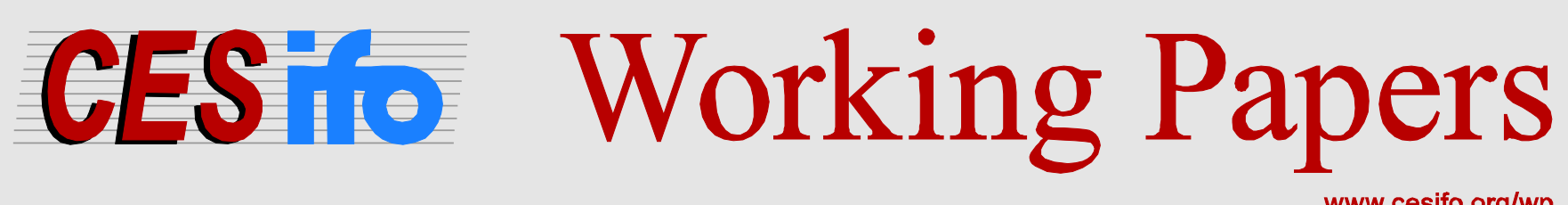

www.cesifo.org/wp

\title{
A Tractable Theory of Choice Based on Cell Behavior
}

\author{
Gonzalo Valdés-Edwards \\ Salvador Valdes-Prieto
}

CESIFO WORKING PAPER NO. 4424

CATEGORY 13: BEHAVIOURAL ECONOMICS

OCTOBER 2013

An electronic version of the paper may be downloaded

- from the SSRN website:

- from the RePEc website:

- from the CESifo website:

wWW.SSRN.com

Www.RePEc.org

www.CESifo-group.org/wp

\section{CESifo}




\title{
A Tractable Theory of Choice Based on Cell Behavior
}

\begin{abstract}
This paper presents a choice model based on a model for the behavior of brain cells that is based on neurological findings. The paper shows that it is possible to define choice as the result of a series of interconnected cellular processes, instead of framing the problem from the point of view of a single optimizing agent. Many of the desirable attributes of the latter are preserved. Under specific conditions identified here, the aggregate behavior of individual brain cells is similar to what a traditional rational optimizing agent would choose. This model is a first step to understand decision-making in terms of cellular interactions.
\end{abstract}

JEL-Code: D870, D810.

Keywords: neuroeconomics, decision-making, economics of neural activity.

Gonzalo Valdés-Edwards*

Pontificia Universidad Católica de Chile

School of Engineering

Santiago / Chile

gvaldese@ing.puc.cl
Salvador Valdes-Prieto

Pontificia Universidad Católica de Chile Economics Institute

Santiago / Chile

svaldes@hacienda.cl

*corresponding author

This draft: October 4, 2013

This paper has benefited from comments from Mark Machina, Andrei Shleifer, Nicola Gennaioli, Ricardo Paredes, René San Martín, and from several participants at the Theory Seminar at the Dept. of Economics, Catholic University of Chile. 


\section{Introduction}

This paper derives decision-making behavior purely from the mechanics of interconnected brain cells, and the behavior of brain cells as documented by the neurological evidence. The main result is that choice can be modeled without assumptions such as the maximization principle and preference axioms. Instead, choice emerges from information processing by brain cells, who receive and send information towards other cells until an action is performed. The model is a first step to understand decision-making in terms of cellular interactions. Moreover, the paper finds that under specific conditions the combined behavior of brain cells is similar to what an optimizing agent would choose.

While classical economics is largely interested in the strategies chosen by rational agents, neuroeconomics analyzes decision-making from a biological perspective (see [1], [2]). Some economists doubt how much they can rely on the present corpus of neuroscience, since its predictive power is still low. More importantly, they wonder how complex behavior (such as market behavior) could be modeled without a general theoretical framework (see [3], [4]).

A growing consensus in neuroscience suggests that the brain makes decisions by following a cycle that starts with the construction of a representation of the problem and the potential actions that can be followed. Later, the courses of actions under consideration are evaluated and one of these is selected. Finally, the outcome observed after the implementation of the selected action is used to improve the quality of future decisions (see [13] for a review). Our model takes this cycle as granted, assuming the brain assigns values to potential actions and later makes a choice over actions.

We model choice according to the following findings: memory retrieval is a slow process (see [14] and [15]). The brain stores prescribed guides for actions (see [16] and [17]), conceives new situations and remembers past experiences (see [18]). The brain feels pleasure in a physical and measurable way (see [19]). Possible rewards are played in the brain before they become realized (see [20]). And a similar calculation to expected utility is performed in the brain (a physical and not as if calculation) (see [21], [22], [23], [24], [25], and [26]).

The individual brain cell model used in this paper combines continuous spike-generation mechanisms and a discontinuous 'after-spike' reset of state variables (see [12] for a review on hybrid spiking models of neurons). This model is not meant to be accurate but sufficiently realistic and simple to permit a tractable model of decision-making based on brain cells. The result is a model where behavior is derived purely from the mechanics of interconnected cells, and yields a tractable set of formulae that summarizes choice.

Although every cell acts independently of one another, our analysis of this model finds that decision-making may be similar to what an optimizing agent would choose. An analogy may be provided by a perfectly competitive market, where each agent and firm chooses independently their consumption and production respectively. Nevertheless, this market allocates resources as if a maximizing agent were present, namely a benevolent planner, as proved by Arrow and Debreu (1954). 
This paper draws on several strands of the literature. One part of the neuroeconomics literature is interested in the conflicts between brain systems, which are treated as if they were rational agents (see [5] for a survey), Another part incorporates some of the physiological constraints faced by the brain in the decision-making process (see [6] for a review). Theoretical neuroscience is concerned with the construction of mathematical and computational models of the brain that characterize what nervous systems do and determine how they function (see [7] for an introduction). Finally, computational neuroscience is interested in the use of computational techniques to model biological neural networks, although it also includes attempts to understand the brain and its functions through theoretical constructs (see [8]). [9] models the process through which the brain maps evidence received from the outside world into decisions, using the standard expected utility approach, and incorporates biological constraints. [10] models choice assuming that "cognitive limitations cause people to focus their attention on some but not all aspects of the world". Similarly, [11] replaces the prior used in classical expected utility with a function that selects "similar" events from memory, instead of taking into account every possible outcome.

Section 2 presents the model in two main steps: first, a general model of a brain cell is presented, and then the model is specialized into three different types of cells, to which a general class of interconnection is added, in order to mimic the three stages in the decision making cycle, in lined with the biological evidence. The mathematics are developed in Appendices. Section 3 presents the main proposition, obtained by analyzing the model. This proposition identifies a set of conditions under which the aggregate behavior of the model is equivalent to the one predicted by expected utility theory. Section 4 summarizes and concludes.

\section{A tractable Neurological Model of Choice}

We model the brain in terms of cells and their connections. The number of cells and connections is a natural number. The model is not meant to be fully accurate since it also aims at tractability.

Cells receive and send information to other cells through connections. Each cell may receive information pulses from other cells or the outside world. Each cell is filled with an inhibitory substance, which acts as brakes that prevent approaching pulses from continuing to the next cells. Although this substance stops information from continuing, the amount of this substance decreases at a rate that depends on the structure of the cell, and the amount and nature of the information received in the different information pulses that arrive. When the amount of inhibitory substance becomes sufficiently small, the cell fires a new pulse towards other cells that are connected with it, by relaxing the sending cell's membrane. This pulse contains processed or modulated information. Different information may be sent towards different cells. The emission of the information pulse allows an immediate fill-up of the cell with inhibitory substance. 
A cell is defined at any moment by the information it holds and the amount of inhibitory substance kept in its interior. Connections are defined by the information pulses sent from one cell to another at a given moment.

Each cell $i$ is a mathematical construct defined at any moment $t$ by:

- Information contained in it, $m_{i} \in \mathbb{R}^{N}$

- Inhibitory substance, $s^{i} \in \mathbb{R}^{+}$

- The maximum amount of inhibitory substance that the cell may retain, $s^{*} \in \mathbb{R}^{+}$

- The rate of decrease of inhibitory substance as it receives information, $v_{i}: \mathbb{R}^{N} \rightarrow \mathbb{R}^{+}$

- How information is processed when sent from cell $i$ to cell $j, w^{i j}: \mathbb{R}^{N} \mathrm{X} \cdots \mathrm{x} \mathbb{R}^{N} \rightarrow \mathbb{R}^{N}$

- The interval of time the cell fires information, $\xi \in \mathbb{R}^{+}$

The state of each connection $i-j$ at time $t$ is defined by:

- The information sent from cell $i$ to cell $j, q_{\text {out }}^{i j} \in \mathbb{R}^{N}$

- The information received by cell $j$ from cell $i, q_{i n}^{i j} \in \mathbb{R}^{N}$

- The amount of time needed for information sent from cell $i$ to cell $j, \varepsilon \in \mathbb{R}^{+}$

- The group of cells that share a common output information channel, $c(i)$, such that whenever one of them fires, all of them are immediately refilled with inhibitory substance.

To understand how cells relate to each other we will need to simulate how outside-world information travels our neural network model. To do so, we will derive $f_{i}(t)$, the frequency with which cell $i$ throws pulses towards other cells. This will allow us to create a tractable model of information processing in the whole neural network.

We model the process experienced by a single cell with the following equations:

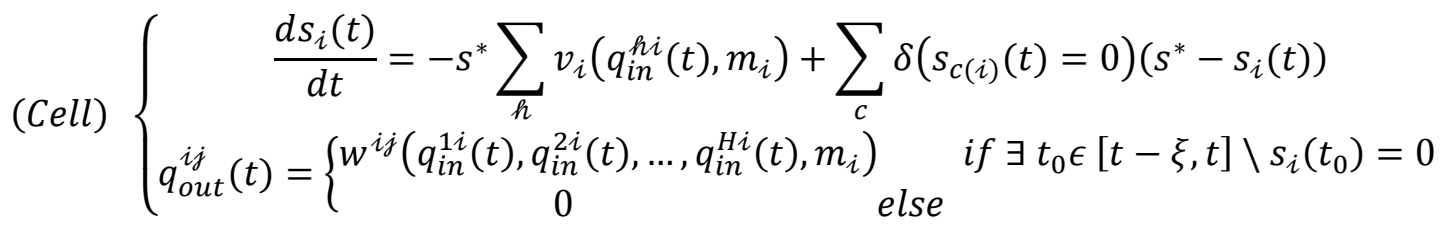

Where $q_{\text {in }}^{\text {hi }}(t)=q_{\text {out }}^{\text {hi }}(t-\varepsilon)$, describes the information pulse sent by cell $h$ to cell $i$, which means that information is not lost, but travels at limited speed.

The rate of decrease of inhibitory substance $v_{i}$ describes how much time is it needed to clear $s^{i}$. When $s^{i}$ becomes zero cell $i$ sends information $q_{\text {out }}^{i j}$ to cell $j$. Finally, $\delta\left(s_{c(i)}(t)=0\right)$ describes the instant at which the cell's membrane is relaxed and is immediately filled with a fixed amount of inhibitory substance, called $s^{*}$. 
The following charts exemplify how the amount of inhibitory substance, $s^{i}(t)$, and one of the possible dimensions of the information pulse $q_{\text {out }}^{i j}(t)$ may evolve in time.
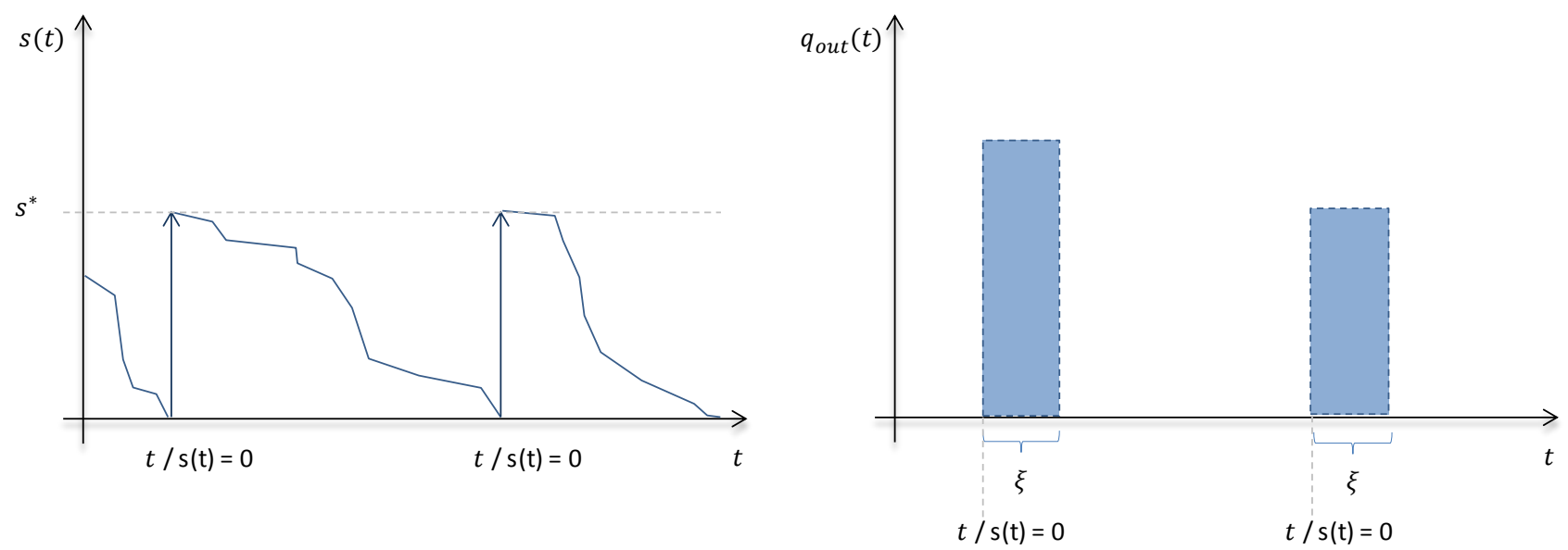

Figure 1: Evolution of inhibitory substance and information pulse over time.

The following sections present models for each step in the choice-making cycle, all of which use the cell model presented here. The architecture of the model is shown below:

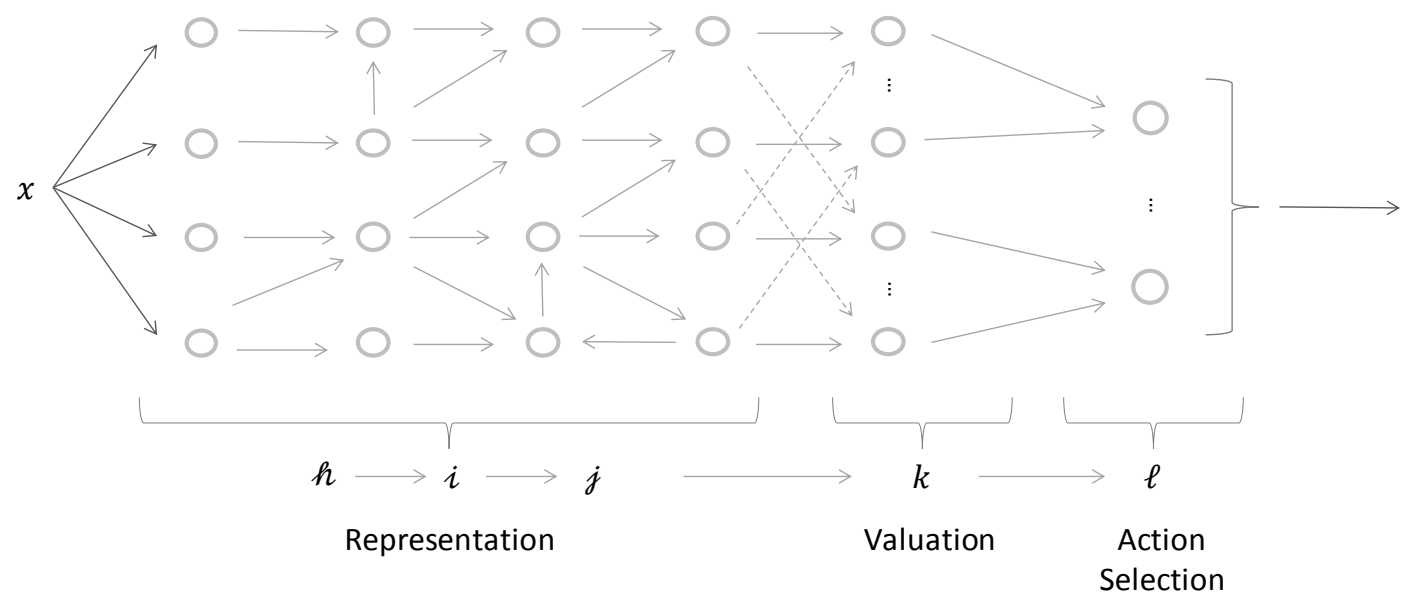

Figure 2: Steps in the decision-making cycle.

The architecture of the model is designed to emulate the decision-making cycle most accepted by neuroscientists. In this model $x$, the information about the world that is felt by the senses, stimulates representation cells. Later on, the final outputs of the representation network (the current situation, potential actions, possible outcomes and memories) are fed into valuation cells. In turn, valuation cells replay memories, reliving pains or pleasures, and send their assessment, together with a suggested action, to action-selection cells. Action-selection cells will "judge" suggested actions in terms of the pains and pleasures involved and send that signal to the nervous central system, which is assumed to execute the action. 


\subsection{Representation}

When new information is sensed, information pulses are fired towards the set of representation cells, which are, in turn, stimulated by these pulses. In particular, cell $i$ stores representation $m_{i} \in \mathbb{R}^{N}$ in a physical form and may sense outside information directly or through other representation cells.

Each representation cell is modeled with the following equations:

(Representation cell) $\begin{cases}\frac{d s_{i}(t)}{d t}=-s_{i}^{*}\left(\sum_{h} v_{i}^{b}\left(q_{i n}^{h i}(t), m_{i}\right)+v_{i}^{o}\left(x_{t}, m_{i}\right)\right)+s_{i}^{*} \delta\left(s_{i}(t)=0\right) \\ q_{\text {out }}^{i j}(t)=\left\{\begin{array}{cc}\sum_{h} w^{i j}\left(q_{i n}^{h i}(t), m_{i}\right) & \text { if } \exists t_{0} \epsilon[t-\xi, t] \backslash s_{i}\left(t_{0}\right)=0 \\ 0 & \text { else }\end{array}\right.\end{cases}$

Where $v_{i}^{b}$ and $v_{i}^{o}$ indicate the rate at which, in representation cell $i$, the inhibitory substance $s_{i}$ diminishes when pulses $q_{h i}$ sent from brain cell $h$ and outside world information $x_{t}$ arrive. Later, whenever $s_{i}$ becomes zero, cell $i$ will fire a pulse equal to $\sum_{h} w_{i j}\left(q_{h i}(t-\varepsilon), m_{i}\right)$ towards cell $j$.

It is important to point out that representation cells may encode all types of memories. For example, some cells may encode whole experiences and others only a few key features. Some cells may record automatic responses (in situation A, perform action B) and others may record situations (in situation $\mathrm{A}$, action $\mathrm{B}$ was performed, and consequence $\mathrm{C}$ occurred). In the next section we assume at least some cells record automatic responses and others record situations.

To calculate the impact of new outside world information let us calculate $f_{i}(t)$, the frequency with which cell $i$ throws pulses towards other cells. Using theorem 1, demonstrated in appendix A, it follows that:

$$
f_{i}(t)=\sum_{h} \xi v_{i}^{b}\left(w_{h i}\left(m_{h}\right), m_{i}\right) f_{h}(t-\varepsilon)+v_{i}^{o}\left(x_{t}, m_{i}\right)
$$

If we use matrices to simplify notation:

$$
f(t)=V^{b} f(t-\varepsilon)+v^{o}\left(x_{t}\right)
$$

And thus:

$$
f(t)=\sum_{n}\left(V^{b}\right)^{n} v^{o}\left(x_{t-n \varepsilon}\right)
$$

Notice that outside-world information may influence events far into the future, depending on the form of matrix $V^{b}$. As an example, if cells are interconnected forming loops, outside-world information will influence representation for a longer amount of time than if those same cells had been connected forming layers. 
Outside-world information travels through the representation system as a whole by changing the firing frequency of neurons. This "information wave" will move at a certain and limited speed. Consequently, in this model decisions that use all available information are time-consuming, and thus, costly. In the following section we assume that, through the process of representation, the brain conceives guides for actions, new situations and remembers past experiences.

\subsection{Valuation}

At this point in the decision making cycle, the agent replays past or imagined memories. The main contribution of valuation cells is to feel how much pain or pleasure a representation evokes.

At time $t$ cell $k$ receives information pulses from two representation cells, $s(k)$ and $a(k) . s(k)$ sends information containing a particular situation $q_{\text {out }}^{s(k), k}$ and $a(k)$ information containing one automatic response $q_{o u t}^{a(k), k}$. When the inhibitory substance in cell $k$ reaches zero it will fire towards cell $\ell$ the automatic response $q_{\text {out }}^{a(k), k}$ and the replayed pleasure of situation $q_{\text {out }}^{s(k), k}, u_{k}\left(q_{\text {out }}^{s(k), k}\right)$.

An adaptation to the general model of a cell yields:

$$
\text { (Valuation Cell) }\left\{\begin{array}{c}
\frac{d s_{k}(t)}{d t}=-s_{k}^{*} v_{k}^{v}\left(q_{i n}^{s(k), k}(t), q_{i n}^{a(k), k}(t)\right)+s_{k}^{*} \delta\left(s_{k}(t)=0\right) \\
q_{\text {out }}^{k \ell}(t)=\left\{\begin{array}{c}
\omega_{k \ell}\left[\begin{array}{c}
q_{i n}^{a(k), k}(t) \\
u_{k}\left(q_{\text {in }}^{s(k), k}(t)\right) \\
0
\end{array}\right] \\
\text { if } \exists t_{k} \in[t-\xi, t] \backslash s_{k}\left(t_{k}\right)=0
\end{array}\right.
\end{array}\right.
$$

where $q_{\text {in }}^{s(k), k}(t)=q_{\text {out }}^{s(k), k}(t-\varepsilon)$ and $q_{\text {in }}^{a(k), k}(t)=q_{\text {out }}^{a(k), k}(t-\varepsilon)$.

Moreover, we define $\omega_{k \ell}$ to be equal to one if cell $k$ sends information towards cell $\ell$ and zero if not, and $v_{k}^{v}$ is the rate at which the inhibitory substance in valuation cell $k$ diminishes.

As will be seen in the following section, $q_{i n}^{a(k), k}$ will be used in the next phase as a suggested actionsignal and $u_{k}\left(q_{i n}^{s(k), k}(t)\right)$ will be used in a calculation similar to the assignment of utils to consequences in standard economic models. Unlike Classical economic models, there might be different valuation functions working at the same time. Namely, $u_{k}\left(q_{i n}^{s(k), k}(t)\right)$ may change with every cell $k$.

The firing frequency of a valuation cell can be calculated using theorem 2, which is demonstrated in appendix B:

$$
f_{k}(t)=\xi^{2} f_{s(k)}(t-\varepsilon) f_{a(k)}(t-\varepsilon) v_{k}^{v}\left(q_{o u t}^{s(k), k}(t-\varepsilon), q_{\text {out }}^{a(k), k}(t-\varepsilon)\right)
$$

The function $v_{k}^{v}$, which is the rate at which the inhibitory substance in cell $k$ diminishes, will affect how much weight the valuation $u_{k}\left(q_{i n}^{s(k), k}\right)$ is given in the decision-making process. As will be seen below, this calculation is very similar to a probability weighting. 


\subsection{Action selection}

In this part of the cycle, each cell behaves independently, and competes in letting their evaluated action-signals pass through. This is because all of these cells send their signals through a single channel. Thus, after any signal passes through, every action-selection membrane will relax and cells will be refilled with inhibitory substance. Therefore, the first evaluated action that is able to pass through the action channel is the one that will be performed by the organism.

The following equations describe the process:

$$
(\text { Action-Selection Cell })\left\{\begin{array}{l}
\frac{d s_{\ell}(t)}{d t}=-s^{*} \sum_{k} v^{a s}\left(q_{i n}^{k \ell}(t)\right)+\sum_{\ell^{\prime}} \delta\left(s_{\ell^{\prime}}(t)=0\right)\left(s^{*}-s_{\ell}(t)\right) \\
q_{\text {out }}^{\ell}(t)=\left\{\begin{array}{cc}
w^{a s}\left(q_{\text {in }}^{k \ell}(t)\right) & \text { if } \exists t_{0} \epsilon[t-\xi, t] \backslash s_{\ell}\left(t_{0}\right)=0 \\
0 & \text { else }
\end{array}\right.
\end{array}\right.
$$

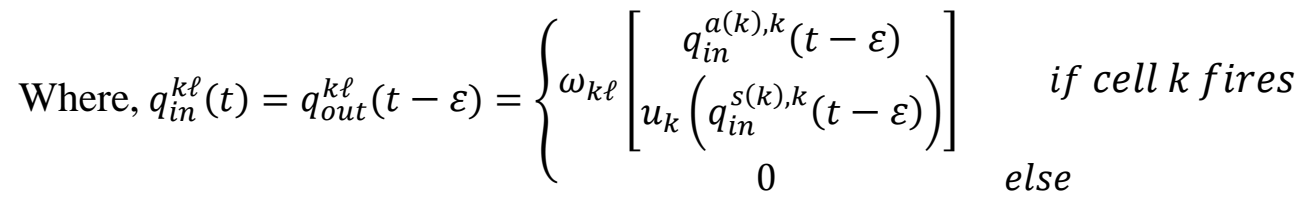

As a simplification, $v^{a s}\left(q_{i n}^{k \ell}(t)\right)=u_{k}\left(q_{i n}^{s(k), k}(t)\right)$, the pain or pleasure signal, and $w^{a s}\left(q_{i n}^{k \ell}(t)\right)=$ $q_{i n}^{a(k), k}(t)$, the automatic response signal (in case cell $k$ fires).

We further simplify the problem by assuming that every valuation cell $k$ that is linked to cell $\ell$ evaluates a unique suggested response. Equivalently, $a(k)=a\left(k^{\prime}\right)=a_{\ell} \Leftrightarrow \omega_{k \ell}=\omega_{k^{\prime} \ell}=1$. A consequence of this simplification is that if a brain with this architecture possesses $\mathrm{N}$ actionselection cells it can ponder in parallel a maximum of $\mathrm{N}$ automatic responses.

If no action cell has "fired" in the time interval of length $d t$ theorem 3, demonstrated in appendix C, asserts that the amount of inhibitory substance in cell $\ell$ will have decreased by

$$
d s_{\ell}(t)=-s^{*} \sum_{k} \omega_{k \ell} \xi f_{k}(t-\varepsilon) u_{k}\left(q_{o u t}^{s(k), k}(t-\varepsilon)\right) d t
$$

The cell whose amount of inhibitory substance reaches zero first, will pass through the suggested action. Later, it will be observed as the action chosen by the organism. If cell $\ell$ 's inhibitory substance reaches zero, it means that since $t_{0}$ (the last time it was filled with inhibitory substance) to the moment it fired $(t)$ the whole substance was absorbed:

$$
-\int_{t_{0}}^{t} d s_{\ell}(t)=s^{*} \Leftrightarrow-\int_{t_{0}}^{t} \frac{d s_{\ell}(t)}{s^{*}}=1
$$

Assume, without loss of generality, that cell $\ell^{*}$ evaluated the performed action. This means that the entire amount of inhibitory substance in cell $\ell^{*}$ was released, and $t^{*}$, the time that cell $\ell^{*}$ needed to do so, was the smallest of all the potential times needed by action-selection cells to do so. 


$$
\left\{\begin{array}{c}
-\int_{t_{0}}^{t^{\ell}} \frac{d s_{\ell}(t)}{s^{*}}=1=\xi \int_{t_{0}}^{t^{\ell}} \sum_{k} \omega_{k \ell} f_{k}(t-\varepsilon) u_{k}\left(q_{\text {out }}^{s(k), k}(t-\varepsilon)\right) d t \\
t^{*}=\min _{\ell}\left(t^{\ell}\right)
\end{array}\right.
$$

This equation says that an action signal is sent by one cell only if it is the first of all action-selection cells to be removed of one hundred percent of its inhibitory substance. This creates a framework in which agents may or may not take action. In this setting there is a clear difference between not reaching a decision (the inhibitory substance never clears) and choosing to do nothing.

Besides, although there is not an optimization process based on preferences, the process of decisionmaking selects one and only one action. As will be seen afterwards, this process can be interpreted as an equivalent of the maximization principle in expected utility if some conditions are met.

\subsection{Understanding the process as a whole}

Here, the three steps are brought together to understand the whole process and how it relates to expected utility theory. The process of decision-making is performed by cells that work independently of one another. Nevertheless it is shown below that if certain conditions are met their aggregate behavior is equivalent to that of a classical optimizing agent.

At the last step, action-taking cell $\ell^{*}$ sends an order to other parts of the body to perform actions. As was shown previously, that order takes into account previous information such that:

$$
\left\{\begin{array}{c}
1=\xi \int_{t_{0}}^{t^{\ell}} \sum_{k} \omega_{k \ell} f_{k}(t-\varepsilon) u_{k}\left(q_{\text {out }}^{s(k), k}(t-\varepsilon)\right) d t \\
t^{*}=\min _{\ell}\left(t^{\ell}\right)
\end{array}\right.
$$

Where $u_{k}\left(q_{\text {out }}^{s(k), k}(t-\varepsilon)\right)$ is the pain or pleasure felt at time $t-\varepsilon$ by cell $k$ and $f_{k}(t-\varepsilon)$ is the rate at which that cell sent that information towards cell $\ell$. In addition, action-taking cell $\ell^{*}$ is the only cell that sends an order, because that order sets every other action-taking cell to a neutral state.

If cell $k$ sends information towards cell $\ell$, then $f_{k}$ is given by:

$$
f_{k}(t)=\xi^{2} f_{s(k)}(t-\varepsilon) f_{a_{\ell}}(t-\varepsilon) v_{k}^{v}\left(q_{\text {out }}^{s(k), k}(t-\varepsilon), q_{\text {out }}^{a_{\ell}, k}(t-\varepsilon)\right)
$$

Where $f_{s(k)}$ and $f_{a_{\ell}}$ are the firing frequencies of cells $s(k)$ and $a_{\ell}$.

Thus, cell $\ell$ sends an order when:

$$
1=\xi^{3} \int_{t_{0}}^{t^{\ell}} \sum_{k} \omega_{k \ell} f_{s(k)}(t-\varepsilon) f_{a_{\ell}}(t-\varepsilon) v_{k}^{v}\left(q_{\text {out }}^{s(k), k}(t-\varepsilon), q_{\text {out }}^{a_{\ell}, k}(t-\varepsilon)\right) u_{k}\left(q_{\text {out }}^{s(k), k}(t-\varepsilon)\right) d t
$$


Next, we need to find the firing frequency of representation cells $s(k)$ and $a_{\ell}$ :

$$
f(t)=\sum_{n}\left(V^{b}\right)^{n} v^{o}\left(x_{t-n \varepsilon}\right)
$$

Where $f$ is the vector of all firing rates of representation cells, $V^{b}$ is a matrix that specifies how the firing frequency of each cell changes when other cells' firing rate change, and $v^{o}$ is a vector that contains how outside world information changes the firing rate of each individual cell. Thus, cells $s(k)$ and $a_{\ell}$ will send information according to that specified rate.

Thus, our complete model of choice is given by:

$$
(\text { Choice })\left\{\begin{array}{c}
f(t)=\sum_{n}\left(V^{b}\right)^{n} v^{o}\left(x_{t-n \varepsilon}\right) \\
1=\xi^{3} \int_{t_{0}}^{t^{\ell}} \sum_{k} \omega_{k \ell} f_{s(k)}(t-\varepsilon) f_{a_{\ell}}(t-\varepsilon) v_{k}^{v}\left(q_{\text {out }}^{s(k), k}(t-\varepsilon), q_{\text {out }}^{a_{\ell}, k}(t-\varepsilon)\right) u_{k}\left(q_{\text {out }}^{s(k), k}(t-\varepsilon)\right) d t \\
t^{*}=\min _{\ell}\left(t^{\ell}\right)
\end{array}\right.
$$

\section{The main proposition}

Analyis of the model of section 2 yields the following:

PROPOSITION 1: The aggregate behavior of cells according to (16) is equivalent to the one predicted by expected utility theory, if and only if the following three conditions are met:

i. Information does not change in the evaluation period and it travels at the same speed (every representation is assessed at the same time and at the same rate).

ii. Every outcome must be evaluated with the same and consistent criterion (experienced utility based on preferences).

iii. Every evaluation must be exclusively based on expected outcomes.

Proof: See Appendix D.

In words, the condition that information does not change in the evaluation period and it travels at the same speed allows us to think of decision-making as if it happened between periods, just like expected utility does. The second condition, that every outcome is evaluated with the same criterion, plays the role of a consistent utility function when evaluating memories. The third condition is the standard assumption that the agent cares about consequences only; this is not so in other choice models (Prospect Theory, for example).

In our model, the first cell that sends the signal of action-taking must have liberated the largest amount of inhibitory substance. The conditions explained in the previous paragraph assure that the 
amount of inhibitory substance released by each action-selection cell changed depending exclusively on future consequences and evaluated each alternative at the same time and with the same criteria. In other words, ex-post, the amount of inhibitory substance that was released by each cell can be interpreted as an expected utility function. Moreover, the cell that released the highest rate of inhibitory substance also sends the signal to be executed as a chosen action, just like a maximizing agent would choose the action that corresponds to the largest utility.

\section{Summary and conclusion}

This paper models the process of decision-making by establishing three different types of cells and a general class of interconnection. This allows the simulation of the three stages of decision-making: representation, valuation, and action-selection. The main assumption of the model is concerned with the processing of information in each stage.

In our model, memory retrieval is a slow process, the brain stores prescribed guides for actions, conceives new situations and remembers past experiences. The brain feels pleasure in a physical and measurable way and possible rewards are played in the brain before they become realized. Finally, a calculation that is similar to expected utility is literally performed by a specialized set of cells.

Analysis of this simple model finds that, under specific conditions, the aggregate behavior of these brain cells is similar to the one of an optimizing agent. However, no cell is optimizing, at least in the standard way. This may be analogous to the hypothetical benevolent planner, which may seem to be governing a perfectly competitive market, although nobody is governing that market.

The assumptions needed for the aggregate behavior of cells to be equivalent to the one predicted by expected utility are there: Information does not change in the evaluation period and it travels at the same speed; Every outcome must be evaluated with the same and consistent criterion; and Every evaluation must be exclusively based on expected outcomes.

This simple model is a first step to connect choice behavior to the biology of cells. Gains from this research line includes understanding how biological constraints on cells, and on cellular interactions, may influence and limit the quality of human decision-making. 


\section{Appendices}

\section{A.- Theorem 1: The average firing rate of a cell affected by individual information pulses}

In this setting, cell two receives impulses from cell one.

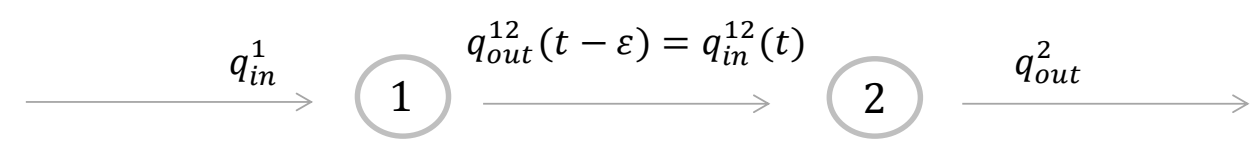

Cellular processes are defined by:

$$
\left(\text { Cell }^{i}\right)\left\{\begin{array}{c}
\frac{d s^{i}(t)}{d t}=-s^{*} v^{i}\left(q_{\text {in }}^{\text {hi }}(t)\right)+s^{*} \delta\left(s^{i}(t)=0\right) \\
q_{\text {out }}^{i j}(t)=\left\{\begin{array}{cl}
w^{i j}\left(q_{\text {in }}^{\text {hi }}(t), m_{i}\right) & \text { if } \exists t_{0} \epsilon[t-\xi, t] \backslash s^{i}\left(t_{0}\right)=0 \\
0 & \text { else }
\end{array}\right.
\end{array}\right.
$$

Where $q_{\text {in }}^{1,2}(t)=q_{\text {out }}^{1,2}(t-\varepsilon)$

The firing rate $f^{i}$ is defined as the number of times cell $i$ fires in a predefined interval of time. According to our model, this is directly related to the number of times the inhibitory substance $s^{i}(t)$ reaches zero. Thus, an approximation for the average firing rate is the average rate of decrease of inhibitory substance, divided by the increase of inhibitory substance after new information is fired.

An approximation of the average firing rate of cell 2 in a $\Delta t$ period of time is:

$$
f^{2}(t, t+\Delta t)=\frac{1}{\Delta t} \int_{t}^{t+\Delta t}-\frac{\frac{d s^{2}}{d t}(u)}{s^{*}} d u=\frac{1}{\Delta t} \int_{t}^{t+\Delta t} v^{2}\left(q_{\text {in }}^{1,2}(u)\right) d u
$$

Now, since information pulses are received from cell 1:

$$
q_{\text {in }}^{1,2}(u)=q_{\text {out }}^{1,2}(u-\varepsilon)=\left\{\begin{array}{cl}
w^{1,2}\left(q_{\text {in }}^{*, 1}(u-\varepsilon)\right) & \text { if } \exists t_{0} \epsilon[u-\varepsilon-\xi, u-\varepsilon] \backslash s^{1}\left(t_{0}\right)=0 \\
0 & \text { else }
\end{array}\right.
$$

Then,

$$
\begin{gathered}
f^{2}(t)=\frac{1}{\Delta t} \int_{t}^{t+\Delta t} v^{2}\left(q_{\text {out }}^{1,2}(u-\varepsilon)\right) d u \\
f^{2}(t)=\frac{1}{\Delta t} \int_{t-\varepsilon}^{t-\varepsilon+\Delta t} v^{2}\left(w^{1,2}\left(q_{\text {in }}^{*, 1}(u)\right)\right) H(u) d u
\end{gathered}
$$

Where $H(u)=\left\{\begin{array}{ll}1 & \text { if } \exists \\ 0 & \text { else }\end{array} t_{0} \epsilon[u-\varepsilon-\xi, u-\varepsilon] \backslash s^{1}\left(t_{0}\right)=0\right.$

In the special case where $q_{\text {in }}^{*, 1}(u)$ is constant at the moment of firing, we find: 


$$
f^{2}(t)=\frac{1}{\Delta t} v^{2}\left(w^{1,2}\left(q_{\text {in }}^{*, 1}(t-\varepsilon, \text { firing })\right)\right) \int_{t-\varepsilon}^{t-\varepsilon+\Delta t} H(u) d u
$$

The last integral can be approximated as the average firing rate of cell 1 in that period multiplied by its firing interval $\xi$ :

Finally, we find:

$$
f^{2}(t)=\frac{1}{\Delta t} v^{2}\left(w^{1,2}\left(q_{\text {in }}^{*, 1}(t-\varepsilon, \text { firing })\right)\right) \Delta t \xi f^{1}(t-\varepsilon)
$$

$$
\begin{gathered}
f^{2}(t)=\xi v^{2}\left(w^{1,2}\left(q_{\text {in }}^{*, 1}(t-\varepsilon, \text { firing })\right)\right) f^{1}(t-\varepsilon) \\
f^{2}(t)=\xi v^{2}\left(q_{\text {out }}^{1,2}(t-\varepsilon, \text { firing })\right) f^{1}(t-\varepsilon)
\end{gathered}
$$

This result is consistent with units: firing rates and $v$ are measured in the inverse of units of time and the firing interval $\xi$ is measured in time units.

\section{B.- Theorem 2: The average firing rate of a cell affected by pairs of information pulses}

In this setting, cell 3 receives impulses from cells 1 and 2.

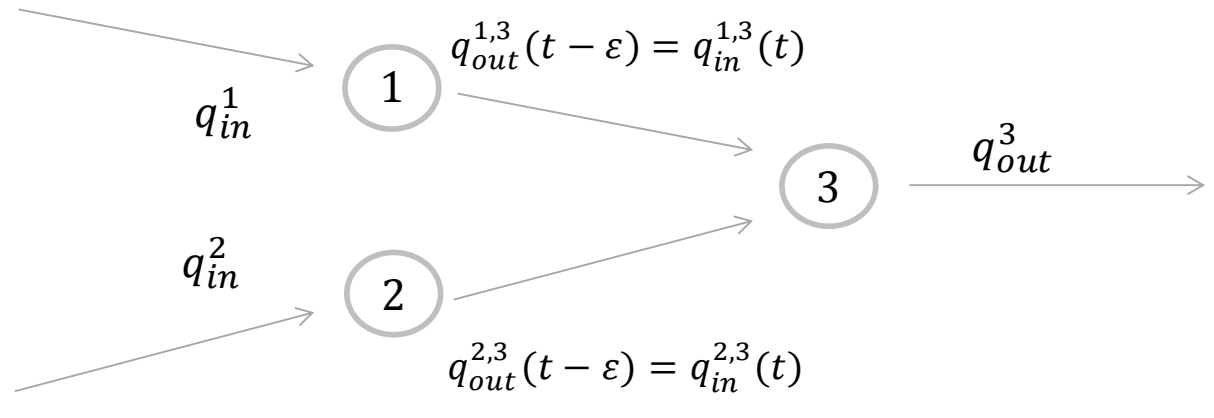

Cellular processes at cell 3 are defined by:

$$
\left\{\begin{array}{c}
\frac{d s_{3}(t)}{d t}=-s^{*} v^{3}\left(q_{\text {in }}^{1,3}(t), q_{\text {in }}^{2,3}(t)\right)+s^{*} \delta\left(s_{3}(t)=0\right) \\
q_{\text {out }}^{3}(t)=\left\{\begin{array}{cc}
w^{3, *}\left(q_{\text {in }}^{1,3}(t), q_{\text {in }}^{2,3}(t)\right) & \text { if } \exists t_{3} \epsilon[t-\xi, t] \backslash s_{3}\left(t_{3}\right)=0 \\
0 & \text { else }
\end{array}\right.
\end{array}\right.
$$

Where $q_{\text {in }}^{1,3}(t)=q_{\text {out }}^{1,3}(t-\varepsilon)$ and $q_{\text {in }}^{2,3}(t)=q_{\text {out }}^{2,3}(t-\varepsilon)$. Besides, $v_{3}$ decreases only if both cells fire information at the same moment.

The firing rate $f^{i}(t)$ is defined as the number of times cell $i$ fires in a given interval. This is directly related to the number of times the inhibitory substance reaches zero. Again, an approximation of the average firing rate is the average rate of decrease of inhibitory substance, divided by the increase of inhibitory substance after new information is fired.

An approximation of the average firing rate of cell 3 in a $\Delta t$ period of time is: 


$$
f^{3}(t)=\frac{1}{\Delta t} \int_{t}^{t+\Delta t}-\frac{\frac{d s^{3}}{d t}(u)}{s^{*}} d u=\frac{1}{\Delta t} \int_{t}^{t+\Delta t} v^{3}\left(q_{\text {in }}^{13}(u), q_{\text {in }}^{23}(u)\right) d u
$$

Now, since information pulses are received from cells 1 and 2:

$$
\begin{aligned}
& q_{\text {in }}^{1,3}(u)=q_{\text {out }}^{1,3}(u-\varepsilon)=\left\{\begin{array}{cl}
w^{1,3}\left(q_{\text {in }}^{1}(u-\varepsilon)\right) & \text { if } \exists t_{1} \epsilon[u-\varepsilon-\xi, u-\varepsilon] \backslash s_{1}\left(t_{1}\right)=0 \\
0 & \text { else }
\end{array}\right. \\
& q_{\text {in }}^{2,3}(u)=q_{\text {out }}^{2,3}(u-\varepsilon)=\left\{\begin{array}{cl}
w^{2,3}\left(q_{\text {in }}^{2}(u-\varepsilon)\right) & \text { if } \exists t_{2} \epsilon[u-\varepsilon-\xi, u-\varepsilon] \backslash s_{2}\left(t_{2}\right)=0 \\
0 & \text { else }
\end{array}\right.
\end{aligned}
$$

The amount of inhibitory substance will decrease only if both cells send pulses. Therefore,

$$
\begin{gathered}
f^{3}(t)=\frac{1}{\Delta t} \int_{t}^{t+\Delta t} v^{3}\left(q_{\text {out }}^{1,3}(u-\varepsilon), q_{\text {out }}^{2,3}(u-\varepsilon)\right) d u \\
f^{3}(t)=\frac{1}{\Delta t} \int_{t-\varepsilon}^{t-\varepsilon+\Delta t} v^{3}\left(w^{1,3}\left(q_{\text {in }}^{*, 1}(u-\varepsilon)\right), w^{2,3}\left(q_{\text {in }}^{*, 2}(u-\varepsilon)\right)\right) H(u) d u
\end{gathered}
$$

Where $H(u)= \begin{cases}1 & \text { if } \exists t_{1}, t_{2} \epsilon[u-\varepsilon-\xi, u-\varepsilon] \backslash s_{1}\left(t_{1}\right)=s_{2}\left(t_{2}\right)=0 \\ 0 & \text { else }\end{cases}$

In the special case where $q_{\text {in }}^{1}$ and $q_{\text {in }}^{2}$ are constant at the moment of firing, we find that:

$$
f^{3}(t)=\frac{1}{\Delta t} v^{3}\left(w^{1,3}\left(q_{i n}^{*, 1}(t-\varepsilon, \text { firing })\right), w^{2,3}\left(q_{i n}^{*, 2}(t-\varepsilon, \text { firing })\right)\right) \int_{t-\varepsilon}^{t-\varepsilon+\Delta t} H(u) d u
$$

The number of times cell 1 fires is its frequency multiplied by $\Delta t$. Thus, the time interval during which cell 1 fires is that number multiplied by its firing time. Similarly, that time multiplied by the firing frequency of cell 2 is the number of times cell 2 fires in that time interval. If we multiply that number by the firing time of cell two we find the total amount of times in which both cells fire together. Thus, we can approximate the last integral as:

$$
\int_{t-\varepsilon}^{t-\varepsilon+\Delta t} H(u) d u=\xi f^{1}(t-\varepsilon) \xi f^{2}(t-\varepsilon) \Delta t
$$

Replacing this result, we find:

$$
\begin{gathered}
f^{3}(t)=\xi^{2} v^{3}\left(w^{1,3}\left(q_{\text {in }}^{*, 1}(t-\varepsilon, \text { firing })\right), w^{2,3}\left(q_{\text {in }}^{*, 2}(t-\varepsilon, \text { firing })\right)\right) f^{1}(t-\varepsilon) f^{2}(t-\varepsilon) \\
f^{3}(t)=\xi^{2} v^{3}\left(q_{\text {out }}^{1,3}(t-\varepsilon, \text { firing }), q_{\text {out }}^{2,3}(t-\varepsilon, \text { firing })\right) f^{1}(t-\varepsilon) f^{2}(t-\varepsilon)
\end{gathered}
$$

This result is consistent with units: firing rates and $v$ are measured in the inverse of units of time and $\xi$ in time units. 


\section{C.- Theorem 3: The average decrease of inhibitory substance in one cell}

We will show that the average decrease of inhibitory substance in a cell that does not fire an information pulse is related to the average firing rate of the previous cell. In this setting, cell two receives impulses from cell one.

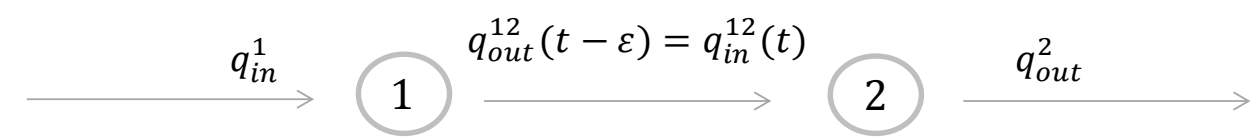

Cellular processes are defined by:

$$
\left(\text { Cell }^{i}\right)\left\{\begin{array}{c}
\frac{d s^{i}(t)}{d t}=-s^{*} v^{i}\left(q_{\text {in }}^{\text {hi }}(t)\right)+s^{*} \delta\left(s^{i}(t)=0\right) \\
q_{\text {out }}^{i j}(t)=\left\{\begin{array}{cl}
w^{i j}\left(q_{\text {in }}^{\text {hi }}(t), m_{i}\right) & \text { if } \exists t_{0} \epsilon[t-\xi, t] \backslash s^{i}\left(t_{0}\right)=0 \\
0 & \text { else }
\end{array}\right.
\end{array}\right.
$$

Where $q_{\text {in }}^{1,2}(t)=q_{\text {out }}^{1,2}(t-\varepsilon)$.

From the first equation, we know that, if cell 2 has not fired, its average decrease in inhibitory substance is given by:

$$
d \bar{s}^{2}(t)=-s^{*} \frac{1}{\Delta t} \int_{t}^{t+\Delta t} v^{2}\left(q_{\text {in }}^{1,2}(u)\right) d u d t
$$

Now, since information pulses are received from cell 1:

$$
q_{\text {in }}^{1,2}(u)=q_{\text {out }}^{1,2}(u-\varepsilon)=\left\{\begin{array}{cl}
w^{1,2}\left(q_{\text {in }}^{*, 1}(u-\varepsilon)\right) & \text { if } \exists t_{0} \epsilon[u-\varepsilon-\xi, u-\varepsilon] \backslash s^{1}\left(t_{0}\right)=0 \\
0 & \text { else }
\end{array}\right.
$$

Then,

$$
\begin{gathered}
d \bar{s}^{2}(t)=-s^{*} \frac{1}{\Delta t} \int_{t}^{t+\Delta t} v^{2}\left(w^{1,2}\left(q_{i n}^{*, 1}(u-\varepsilon)\right)\right) d u d t \\
d \bar{s}^{2}(t)=-s^{*} \frac{1}{\Delta T} \int_{t-\varepsilon}^{t-\varepsilon+\Delta t} v^{2}\left(w^{1,2}\left(q_{i n}^{*, 1}(u)\right)\right) H(u) d u d t
\end{gathered}
$$

Where $H(u)= \begin{cases}1 & \text { if } \exists \\ 0 & \text { else }\end{cases}$

In the special case where $q_{\text {in }}^{*, 1}$ is constant at the moment of firing, we find:

$$
d \bar{s}^{2}(t)=-s^{*} \frac{1}{\Delta t} v^{2}\left(w^{1,2}\left(q_{i n}^{*, 1}(t-\varepsilon, \text { firing })\right)\right) \int_{t-\varepsilon}^{t-\varepsilon+\Delta t} H(u) d u d t
$$


The last integral can be approximated as the average firing rate of cell 1 in that period multiplied by its firing length:

Finally, we find:

$$
d \bar{s}^{2}(t)=-s^{*} \frac{1}{\Delta t} v^{2}\left(w^{1,2}\left(q_{\text {in }}^{*, 1}(t-\varepsilon, \text { firing })\right)\right) \Delta t \xi f^{1}(t-\varepsilon) d t
$$

$$
\begin{gathered}
d \bar{s}^{2}(t)=-s^{*} \xi v^{2}\left(w^{1,2}\left(q_{\text {in }}^{*, 1}(t-\varepsilon, \text { firing })\right)\right) f^{1}(t-\varepsilon) d t \\
d \bar{s}^{2}(t)=-s^{*} \xi v^{2}\left(q_{\text {out }}^{1,2}(t-\varepsilon, \text { firing })\right) f^{1}(t-\varepsilon) d t
\end{gathered}
$$

This is consistent with units: $d \bar{s}^{2}$ and $s^{*}$ is measured in amount of inhibitory substance, firing rates and $v$ are measured in the inverse of units of time and $\xi$ and $d t$ are measured in time units.

\section{D.- PROPOSITION 1: Equivalency between choice based on cell behavior and Expected Utility}

We show that our model of choice based on cell behavior can be interpreted as a maximization of expected utility if the following conditions are met:

1. Information does not vary over the period of evaluation and every representation influences action-selection cells through valuation cells at the same moment. This means that matrix $V^{b}$ is such that representation cells that send information to valuation cells do so at the same moment and the information flow is constant over time.

2. Every outcome must be evaluated with the same and consistent criterion (experienced utility based on preferences). This means $u_{k}()=.u($.$) for all cells k$.

3. Every evaluation is exclusively based on expected outcomes. This means that $q_{\text {out }}^{s(k), k}$ is always a consequence and never a previous experience.

Part I: If the above conditions are met, then the model is equivalent to a utility maximizing agent.

The equation that describes the moment at which cell $\ell$ fires is given by:

$$
1=\xi \int_{t_{0}}^{t^{\ell}} \sum_{k} \omega_{k \ell} f_{k}(t-\varepsilon) u_{k}\left(q_{\text {out }}^{s(k), k}(t-\varepsilon)\right) d t
$$

Condition 1 means that the rate at which inhibitory substance decreases is constant. Thus:

$$
\int_{t_{0}}^{t^{\ell}} \sum_{k} \omega_{k \ell} f_{k}(t-\varepsilon) u_{k}\left(q_{\text {out }}^{s(k), k}(t-\varepsilon)\right) d t=\left(t^{\ell}-t_{0}\right) \sum_{k} \omega_{k \ell} f_{k} u_{k}\left(q_{\text {out }}^{s(k), k}\right)
$$


Where $t^{\ell} \in\left[t_{0}+\xi, t_{0}\right]$ is the time at which cell $\ell$ would have fired a signal describing an automatic response if it were the first action-selection cell to do so. Then,

$$
t^{\ell}=t_{0}+\frac{1}{\xi} \frac{1}{\sum_{k} \omega_{k \ell} f_{k} u_{k}\left(q_{o u t}^{s(k), k}\right)}
$$

It follows that, if $t^{*}=\min _{\ell}\left(t^{\ell}\right)$, then $\left(q_{\text {out }}^{a_{\ell}, k}\right)^{*}$, the automatic response performed, must maximize the denominator, so:

$$
\left(q_{\text {out }}^{a_{\ell}, k}\right)^{*}=\underset{m_{a_{\ell}}}{\operatorname{argmax}} \sum_{k} \omega_{k \ell} f_{k} u_{k}\left(q_{\text {out }}^{s(k), k}\right)
$$

We can expand and re-write this last equation as:

$$
m_{a_{\ell}}{ }^{*}=\underset{m_{a_{\ell}}}{\operatorname{argmax}} \sum_{k} \omega_{k \ell} f_{s(k)} f_{a_{\ell}} v_{k}^{v}\left(q_{\text {out }}^{s(k), k}, q_{\text {out }}^{a_{\ell}, k}\right) u_{k}\left(q_{\text {out }}^{s(k), k}\right)
$$

Besides, if $u_{k}\left(q_{\text {out }}^{s(k), k}\right)=u\left(q_{\text {out }}^{s(k), k}\right)$ (condition 2), and $q_{\text {out }}^{s(k), k}$ is always a consequence (condition 3), our formula becomes the equivalent of a linear transformation of an expected utility.

In particular, the functional form of the subjective probability measure used to value expectations is of the form:

$$
P\left(q_{\text {out }}^{s(k), k} / q_{\text {out }}^{a_{\ell}, k}\right)=\frac{\omega_{k \ell} f_{s(k)} f_{a_{\ell}} v_{k}^{v}\left(q_{\text {out }}^{s(k), k}, q_{\text {out }}^{a_{\ell}, k}\right)}{\sum_{k} \omega_{k \ell} f_{s(k)} f_{a_{\ell}} v_{k}^{v}\left(q_{\text {out }}^{s(k), k}, q_{\text {out }}^{a_{\ell}, k}\right)}
$$

Part II: If the above conditions are met, then the model is equivalent to a utility maximizing agent.

The model shows actions $q_{\text {out }}^{a_{\ell}, k}$ can be ordered as in preferences in terms of $t^{\ell}$, the time they need to clear the inhibitory substance in action-selection cell $\ell$ (remember there is only one action per action-selection cell). Then: $q_{\text {out }}^{a_{\ell}, k} \geqslant q_{\text {out }}^{a_{\ell,}, k} \Leftrightarrow t^{\ell} \leq t^{\ell^{\prime}}$.

Besides, $t^{\ell}$ is defined by the formula:

$$
1=\xi^{3} \int_{t_{0}}^{t^{\ell}} \sum_{k} \omega_{k \ell} f_{s(k)}(t-\varepsilon) f_{a_{\ell}}(t-\varepsilon) v_{k}^{v}\left(q_{\text {out }}^{s(k), k}(t-\varepsilon), q_{\text {out }}^{a_{\ell}, k}(t-\varepsilon)\right) u_{k}\left(q_{\text {out }}^{s(k), k}(t-\varepsilon)\right) d t
$$

The last formula can be re-written as follows (terms a to e): 


$$
\begin{gathered}
\frac{1}{\xi^{3}}=\left(t^{\ell}-t_{0}\right)\left[\sum_{k / q_{\text {out }}^{s(k), k} \in C} \bar{g}_{a_{\ell}}\left(q_{\text {out }}^{s(k), k}\right) \bar{u}\left(\bar{q}_{\text {out }}^{s(k), k}\right)\right] \\
+\left(t^{\ell}-t_{0}\right)\left[\sum_{k} \bar{g}_{a_{\ell}}\left(q_{\text {out }}^{s(k), k}\right) \bar{u}\left(\bar{q}_{\text {out }}^{s(k), k}\right)-\sum_{k / q_{\text {out }}^{s(k), k} \in C} \bar{g}_{a_{\ell}}\left(q_{\text {out }}^{s(k), k}\right) \bar{u}\left(\bar{q}_{\text {out }}^{s(k), k}\right)\right] \\
+\left(t^{\ell}-t_{0}\right)\left[\sum_{k} \bar{g}_{a_{\ell}}\left(q_{\text {out }}^{s(k), k}\right)\left(u_{k}\left(\bar{q}_{\text {out }}^{s(k), k}\right)-\bar{u}^{\left(\bar{q}_{\text {out }}^{s(k), k}\right)}\right)\right] \\
+\left[\sum_{k} \int_{t_{0}}^{t^{\ell}} \bar{g}_{a_{\ell}}\left(q_{\text {out }}^{s(k), k}\right)\left(u_{k}\left(q_{\text {out }}^{s(k), k}(t-\varepsilon)\right)-u_{k}\left(\bar{q}_{\text {out }}^{s(k), k}\right)\right) d t\right] \\
+\left[\sum_{k} \int_{t_{0}}^{t^{\ell}} u_{k}\left(q_{\text {out }}^{s(k), k}(t-\varepsilon)\right)\left(g_{a_{\ell}}\left(t, q_{\text {out }}^{s(k), k}\right)-\bar{g}_{a_{\ell}}\left(q_{\text {out }}^{s(k), k}\right)\right) d t\right]
\end{gathered}
$$

Where

$$
\left\{\begin{array}{c}
g_{a_{\ell}}\left(t, q_{\text {out }}^{s(k), k}\right)=\omega_{k \ell} f_{s(k)}(t-\varepsilon) f_{a_{\ell}}(t-\varepsilon) v_{k}^{v}\left(q_{\text {out }}^{s(k), k}(t-\varepsilon), q_{\text {out }}^{a_{\ell}, k}(t-\varepsilon)\right) \\
\bar{g}_{a_{\ell}}\left(q_{\text {out }}^{s(k), k}\right)=\frac{1}{\left(t^{\ell}-t_{0}\right)} \int_{t_{0}}^{t^{\ell}} g_{a_{\ell}}\left(t, q_{\text {out }}^{s(k), k}\right) d t \\
u_{k}\left(\bar{q}_{\text {out }}^{s(k), k}\right)=\frac{1}{\left(t^{\ell}-t_{0}\right)} \int_{t_{0}}^{t^{\ell}} u_{k}\left(q_{\text {out }}^{s(k), k}(t-\varepsilon)\right) d t \\
\bar{u}\left(\bar{q}_{\text {out }}^{s(k), k}\right)=\frac{1}{K} \sum_{k} u_{k}\left(\bar{q}_{\text {out }}^{s(k), k}\right) \\
C \text { is the set of situations that consists only of consequences }
\end{array}\right.
$$

Thus, $t^{\ell}$ will become a transformation of a expected utility function only if the last four terms in the equation are always equal to zero. Terms e) and d) represent condition 1, term c) represents condition 2, and term b) represents condition 3 . 


\section{References}

[1] C. Camerer, G. Loewenstein and D. Prelec, "Neuroeconomics: How Neuroscience Can Inform Economics," Journal of Economic Literature, vol. 43, pp. 9-64, 2005.

[2] J. Vromen, "Neuroeconomics: two camps gradually converging: what can economics gain from it?," International Review of Economics, vol. 58, no. 3, pp. 267-285, 2011.

[3] G. Harrison, "Neuroeconomics: A Critical Reconsideration," Economics and Philosophy, vol. 24, no. 3, pp. 303-344, 2008.

[4] P. Kenning and H. Plassmann, "NeuroEconomics: An overview from an economic perspective," Brain Research Bulletin, vol. 67, pp. 343-354, 2005.

[5] I. Brocas and J. Carrillo, "Dual-process theories of decision-making: a selective survey, working paper," Journal of Economic Psychology.

[6] P. Glimcher, M. Dorris and H. Bayer, "Physiological utility theory and the neuroeconomics of choice," Games and Economic Behavior, vol. 52, no. 2, pp. 213-256, 2005.

[7] P. Dayan and L. Abbott, Theoretical Neuroscience. Computational and Mathematical Modeling of Neural Systems, MIT Press, 2005.

[8] M. Arbib, The Handbook Of Brain Theory And Neural Networks, Second Edition, MIT Press, 2002.

[9] I. Brocas and J. Carrillo, "From perception to action: An economic model of brain processes," Games and Economic Behavior, vol. 75, no. 1, pp. 81-103, 2012.

[10] P. Bordalo, N. Gennaioli and A. Shleifer, "Salience Theory of Choice under Risk," NBER working paper 16387, 2010.

[11] I. Gilboa and D. Schmeidler, "Utility in case-based decision theory," Journal of Economic Theory, vol. 105, no. 2, pp. 483-502, 2002.

[12] E. M. Izhikevich, "Hybrid spiking models," Philosophical Transactions of the Royal Society, vol. 368, p. 5061-5070, 2010.

[13] A. Rangel, C. Camerer and P. Montague, "A framework for studying the neurobiology of value-based decision making," Nature reviews, Neuroscience, vol. 9, pp. 1-13, 2008.

[14] L. Carrier and H. Pashler, "Attentional Limits in Memory Retrieval," Journal of Experimental Psychology: Learning, Memory and Cognition, vol. 21, no. 5, pp. 1339-1348, 1995.

[15] J. Schall, "Neural Correlates of Decision Processses: Neural and Mental Chronometry," Current Opinion in Neurobiology, vol. 13, no. 2, pp. 182-186, 2003.

[16] S. A. Bunge, "How we use rules to select actions: A review of evidence from cognitive neuroscience," Cognitive, Affective, \& Behavioral Neuroscience, vol. 4, no. 4, pp. 564-579, 2004.

[17] G. Morris, A. Nevet, D. Arkadir, E. Vaadia and H. Bergman, "Midbrain dopamine neurons encode decisions for future action," Nature Neuroscience, vol. 9, pp. 1057-1063, 2006.

[18] R. Buckner, "The role of Hippocampus in prediction and imagination," Annual review of psychology, vol. 61, pp. 27-48, 2010.

[19] S. Leknes and I. Tracey, "A common neurobiology for pain and pleasure," Nature reviews, vol. 9, pp. 314-320, 2008. 
[20] K. Samejima, Y. Ueda, K. Doya and M. Kimura, "Representation of action-specific reward values in the striatum," Science, no. 310, p. 1337-1340, 2005.

[21] H. Plassmann, J. O’Doherty and A. Rangel, "Orbitofrontal Cortex Encodes Willingness to Pay in Everyday Economic Transactions," The Journal of Neuroscience, vol. 27, no. 37, p. 9984 9988, 2007.

[22] J. Kable and P. Glimcher, "The neural correlates of subjective value during intertemporal choice," Nature Neuroscience, vol. 10, pp. 1625-1633, 2007.

[23] B. Knutson, J. Taylor, M. Kaufman, R. Peterson and G. Glover, "Distributed Neural Representation of Expected Value," The Journal of Neuroscience, vol. 25, no. 19, pp. 48064812, 2005.

[24] M. Symmonds, P. Bossaerts and R. Dolan, "A behavioral and Neural Evaluation of Prospective Decision-Making under Risk," The Journal of Neuroscience, vol. 30, no. 43, pp. 14380-14389, 2010.

[25] N. Camille, C. Griffiths, K. Vo, L. Fellows and J. Kable, "Ventromedial Frontal Lobe Damage Disrupts Value Maximization in Humans," The Journal of Neuroscience, vol. 31, no. 20, pp. 7527-7532, 2011.

[26] M. Kang, A. Rangel, M. Camus and C. Camerer, "Hypothetical and Real Choice Differentially Activate Common Valuation Areas," The Journal of Neuroscience, vol. 31, no. 2, pp. 461-468, 2011. 